\title{
Aortic Valve and Root Replacement for an adolescent with Sickle Cell Disease, Hodgkin's Lymphoma and History of Cerebrovascular Accident.
}

\author{
AHMED ELMAHROUK ${ }^{1}$, Hani Barnawi², Hassan Alshehri ${ }^{1}$, Majed Almutairi ${ }^{1}$, Ghadeer \\ Mokhtar $^{1}$, Ahmed Jamjoom ${ }^{3}$, and Uthman Aluthman ${ }^{1}$ \\ ${ }^{1}$ King Faisal Specialist Hospital and Research Centre - Jeddah \\ ${ }^{2}$ King Saud bin Abdulaziz University for Health Sciences \\ ${ }^{3}$ Affiliation not available
}

May 14, 2020

\begin{abstract}
Background: Sickle cell anemia is an autosomal recessive inherited disorder that affects approximately $5 \%$ of the world population. These patients are at greater risk for developing Hodgkin's lymphoma. Cardiopulmonary bypass can trigger lethal vaso-occlusive crises in those patients if they are subjected to hypoxia, hypothermia, acidosis, or low-flow states. Case presentation: This case report describes a patient with sickle cell anemia and history of stroke was diagnosed with Bicuspid aortic valve stenosis and aneurysmal dilatation of the ascending aorta complicated with infective endocarditis. During routine workup he was discovered to have Hodgkin's Lymphoma. He successfully underwent mechanical aortic valve and aortic root replacement. He underwent exchange transfusion preoperatively and one time immediately before initiating of Cardiopulmonary bypass. There was no major vaso-occlusive crisis occurred throughout the surgery. Patient was discharged in stable condition, and was scheduled for involved site radiation therapy for Hodgkin's Lymphoma management. Conclusion: Sickle Cell Disease can be very challenging during cardiopulmonary bypass. Exchange transfusion can reduce HbS, and increase hematocrit level. Mild hypothermia can be used if sufficient CPB flows and venous saturation are maintained.
\end{abstract}

\section{Case presentation:}

This case report describes a patient with sickle cell anemia and history of stroke was diagnosed with Bicuspid aortic valve stenosis and aneurysmal dilatation of the ascending aorta complicated with infective endocarditis. During routine workup he was discovered to have Hodgkin's Lymphoma. He successfully underwent mechanical aortic valve and aortic root replacement. He underwent exchange transfusion preoperatively and one time immediately before initiating of Cardiopulmonary bypass. There was no major vaso-occlusive crisis occurred throughout the surgery. Patient was discharged in stable condition, and was scheduled for involved site radiation therapy for Hodgkin's Lymphoma management.

\section{Conclusion:}

Sickle Cell Disease can be very challenging during cardiopulmonary bypass. Exchange transfusion can reduce $\mathrm{HbS}$, and increase hematocrit level. Mild hypothermia can be used if sufficient CPB flows and venous saturation are maintained.

\section{Introduction:}

Sickle cell anemia is an autosomal recessive inherited disorder that affects approximately $5 \%$ of the world population. It is commonly prevalent among African Americans. However, it is also seen in Southern Europe, 
India, and the Middle East [1,2]. Sickle Cell Disease (SCD) is characterized by its recurrent vaso-occlusive crises which may lead to multi-organ damage and reduction in survival rate [3]. These patients are at greater risk for developing malignancies especially hematological malignancy, particularly Hodgkin's lymphoma [4], which has a great impact on perioperative management.

Advanced cardiopulmonary disorder has appeared as a leading cause of death [5] with improvements in diagnostic and surgical techniques along with anesthetic management skills, there is a substantial increase in patients with SCD who undergo cardiac surgery [2].

Patients with SCD especially the homozygous type, whom are assigned for cardiac surgery are at greater risk for potentially lethal vaso-occlusive crises that can be triggered by hypoxia, hypothermia, acidosis, or low-flow states. All of which can be provoked by cardiopulmonary bypass (CPB), which may increase the likelihood of significant postoperative complications [1].

\section{Case report:}

A 20 years old male, a known case of SCD, with history of stroke with minimal right-side weakness. He was diagnosed with aortic valve stenosis under conservative management. The patient was referred to our center as a case of acute infective endocarditis, bicuspid aortic valve with sub aortic membrane stenosis. His main complaint was fever otherwise no other symptoms (no chest pain, no shortness of breath, no nausea, vomiting or change in bowel habits, no history of contact with an ill person or traveling, no contact with animal or raw milk ingestion)

On examination: Patient was conscious, oriented and hemodynamically stable. Chest was clear on auscultation, with normal oxygen saturation on room air. The patient was febrile with temperature $>38 \mathrm{C}$.

Precordial auscultation showed harsh ejection systolic murmur with crescendo-decrescendo configuration, medium pitch with grade III intensity, best heard over the aortic area radiated to carotid.

Blood test, showed $\mathrm{Hb}$ of $7.4 \mathrm{~g} / \mathrm{dl}$, HBs 92.2 , WBC of 21, three blood cultures from different sites were negative. Q-fever, Brucella and Dengue fever workup were negative. The patient was started on empirical IV antibiotics ceftriaxone and vancomycin.

Trans-thoracic echocardiography showed severe concentric hypertrophy, normal left ventricular (LV) function with LV ejection fraction of $60 \%$. Aortic valve (AV) was bicuspid with no evidence of vegetation, ascending aortic root dilation $4.1 \mathrm{~cm}$. Trans-esophageal echocardiographyshowed thickened and restricted AV leaflets, there was severe aortic valve stenosis with peak and mean gradients of 160 and $90 \mathrm{mmHg}$ respectively. There was no vegetation or aortic root abscesses. (Figure $1 \mathrm{a}, \mathrm{b}, \mathrm{c}$ ).

CT chest and abdomen: was done looking for a source of infection, it showed generalized lymph node enlargement in the axilla, abdomen, and inguinal area. An excisional biopsy of left inguinal LN $\left(3^{*} 2 \mathrm{~cm}\right)$ was sent for histopathology and showed Hodgkin lymphoma (Figure 3).

Brain CT + CT Angiogram: Re-demonstration of multiple old infractions mainly seen in the occipital lobe, posterior limb of the right internal capsule and the corona radiata/centrum semiovale with encephalomalacia changes. No evidence of acute infraction. No acute hemorrhage (Figure 2). CT angiography was unremarkable.

MRI: was done and showed multiple old infarctions predominantly in the occipital region and the corona radiata/centrum.

PET scan (was done after the surgery): Multiple FDG avid enlarged lymph nodes seen in the left inguinal, obturator and external iliac lymph node chain. The largest measures around $3.2 \mathrm{~cm}$ (SUV max 15). The urinary bladder demonstrates irregular outlines with trabeculation (Figure 4). Evidence of FDG avid adenopathy in the pelvis, as detailed above in keeping with history of lymphoma. Post-operative activity in the mediastinum and myocardium/pericardium, for clinical correlation. Abnormal appearance of the urinary bladder for clinical correlation. 
The patient was scheduled for AV and ascending aorta replacement.

Cardiopulmonary bypass (CPB) management: The decision was made to perform an exchange transfusion using packed red blood cells (PRBCs) in the pre-operative period and immediately before initiation of CPB.

The patient's height was $160 \mathrm{~cm}$ and weight $40 \mathrm{~kg}$, body surface area of $1.33 \mathrm{~m} 2$ and his hemoglobin level was $6.35 \mathrm{~g} / \mathrm{L}$.

The circuit was adapted for the exchange transfusion by adding a 1/4-3/8-3/8" Y connector within the venous line. The two $3 / 8$ " ends of the $\mathrm{Y}$ were connected to the standard venous line of the circuit. The $1 / 4$ " end of the $\mathrm{Y}$ was connected to $1 / 4$ " tubing and attached to a separate cardiotomy reservoir (cell saver).

Circuit was primed with $900 \mathrm{ml}$ of crystalloid fluid. After that the crystalloid fluid was replaced with 250 $\mathrm{ml}$ of $5 \%$ albumen, $50 \mathrm{mEq}$ of sodium bicarbonate, 10,000 units of heparin, $2 \mathrm{~g}$ of cefazolin, and $100 \mathrm{ml}$ of $5 \%$ mannitol. After the circuit was primed, the excess crystalloid fluid was drained from the circuit, and 4 units of PRBCs were added to the venous reservoir.

Once the chest was opened and the heart was exposed, the patient was systemically heparinized. An $18 \mathrm{Fr}$ aortic cannula was placed in the ascending aorta. Venous drainage was accomplished through straight cannulation with $32 / 40 \mathrm{Fr}$ double stage venous cannula placed in the right atrium. Once an activated clotting time of 371 seconds was achieved, the exchange transfusion was initiated. With the venous line clamped distal to the Y connector, the line to the separate cardiotomy reservoir was opened, allowing the patient's blood to drain. At the same time, blood from CPB machine was transfused to the patient to maintain the patient hemodynamically stable. After an exchange transfusion of $1500 \mathrm{~mL}$ was achieved, the patient was placed on $\mathrm{CPB}$.

Normal hypothermia was used, and the patient was cooled to a bladder temperature of $34^{\circ} \mathrm{C}$. Antegrade cardioplegia cannulas were placed. An aortic cross clamp was placed, and the heart was initially arrested with antegrade cold-crystalloid. A St. Jude mechanical aortic valve size 21-mm was implanted and ascending aorta was replaced with vaxcotec graft size $26 \mathrm{~mm}$. The CPB flows were maintained at a minimum cardiac index of $2.4 \mathrm{~L} / \mathrm{min}$. Venous saturation was kept $>70 \%$ and no acidosis occurred during the CPB period. Two units of PRBCs were given during the bypass run. Hemoglobin level was $10.6 \mathrm{~g} / \mathrm{L}$. The patient was re-warmed to a bladder temperature of $36^{\circ} \mathrm{C}$. The aortic cross-clamp was removed after 134 minutes. After resumption of normal sinus rhythm, the patient was ventilated and successfully weaned from $\mathrm{CPB}$. Total CPB time was 163minutes. There were surgical oozing with no obvious site of bleeding, multiple rounds of blood products (FFP, PRBCs) were given and decision was made to leave the chest opened and transfer to the intensive care unit (ICU).

In the ICU, the patient required surgical re-exploration for hemostasis with no site for bleeding, and another round of blood product were given, and bleeding stopped. Chest was closed on the second postoperative day and the patient was extubated. (6)

All chest tubes were removed in timely fashion, his general condition continued to improve and he was discharged in a stable condition. Follow up appointments with oncology and hematology outpatients' clinics were given to continue with management. In view of his early stage Hodgkin's Lymphoma and absent B symptoms (fever, night sweats, and weight loss), involved site radiation therapy (ISRT) up to 36 Gy as a single Modality.

Recently, the patient was admitted through the ER as a case of acute right occipital ischemic stroke. Regarding cardiac point of view, there were no complaints and he showed a significant improvement in the symptoms. There was no major surgical complication. The admission duration was 2 weeks after which he was discharged in a stable condition.

\section{Discussion:}


This case presents a number of challenges that we had to tackle, one of which was vaso-occlusive crisis during aortic root replacement which is considered one of the major surgeries. To overcome this, our surgical plan included:

Exchange transfusion, which can aid in two ways, one is by reducing HbS, and the other is by increasing the preoperative hematocrit level. Thus, facilitating a better oxygen delivery to the tissues. [2,7] This measure was taken preoperatively and reduced the $\mathrm{HbS}$ to 73.5. Also, perioperative exchange transfusion was done to reduce the vaso-occlusive Crisis.

Mild hypothermia $\left(>32^{\circ} \mathrm{C}\right)$ is traditionally used for myocardial preservation by decreasing the metabolic rate and reducing the oxygen demand. However, hypothermia in patients with sickle cell anemia is still controversial with almost half of the published reports' patients were maintained on normothermia during $\mathrm{CPB}$, the rest underwent a mild hypothermia $\left(>32^{\circ} \mathrm{C}\right) .[1]$

ln our case, aiming at minimizing the risk of vaso-occlusive crisis, we performed a mild hypothermia of $34^{\circ} \mathrm{C}$ during the surgery, $\mathrm{CPB}$ flows were maintained at a minimum cardiac index of $2.4 \mathrm{~L} / \mathrm{min}$, venous saturation was kept $>70 \%$, and no acidosis occurred during the CPB period. There was no major vaso-occlusive crisis occurred throughout the surgery

Another challenge is mechanical vs biological valve. Even though mechanical valves are superior in durability, they are linked with an increased risk of thrombosis, hemolysis, sickle cell crisis, and lifelong anticoagulation use, which can predispose the patient to hemorrhagic complications. [5] [1] In our case, when measuring the risk and benefits of both types and due to his young age, we prioritized the use of a mechanical valve.

\section{Conclusions:}

Sickle Cell Disease can be very challenging during cardiopulmonary bypass. Vaso-occlusive crises can be triggered by hypoxia, hypothermia, acidosis, or low-flow states. Exchange transfusion can reduce HbS, and increase hematocrit level, this will facilitate a better oxygen delivery. Mild hypothermia can be used if sufficient CPB flows are maintained at a minimum cardiac index of $2.4 \mathrm{~L} / \mathrm{min}$ and venous saturation is kept more than $70 \%$.

\section{References:}

1. Silveira LMVD, Tagliari AP, Costa RDD, Martins CB and Wender O.: Aortic Valve Replacement Combined with Ascending Aortic Aneurysmectomy in a Patient with Sickle Cell Disease: a Case Report. Braz J Cardiovasc Surg . 2018; 33(2):203-205. doi:10.21470/1678-9741-2017-0185

2. Yousafzai SM, Ugurlucan M, Al Radhwan OA, Al Otaibi AL and Canver CC: Open heart surgery in patients with sickle cell hemoglobinopathy. Circulation. 2010; 121 : 14-19.

3. Edwin F, Aniteye E, Tettey M, Tamatey M, Entsua-Mensah K, Ofosu-Appiah E, Sereboe L, Gyan B, Adzamli I and Frimpong-Boateng K: Hypothermic cardiopulmonary bypass without exchange transfusion in sickle-cell patients: a matched-pair analysis. Interactive cardiovascular and thoracic surgery. 2014; 19(5):771-776.

4. Moutaouekkil el M, Najib A, Ajaja R, Arji M and Slaoui A.: Heart valve surgery in patients with homozygous sickle cell disease: A management strategy. Ann Card Anaesth . 2015;18(3):361-366.

5. Crawford TC, Carter MV, Patel RK, Suarez-Pierre A1, Lin SZ1, Magruder JT1, Grimm JC1, Cameron DE1, Baumgartner WA1 and Mandal K1: Management of sickle cell disease in patients undergoing cardiac surgery. J Card Surg . 2017; 32(2):80-84.

6. Elmahrouk AF, Ismail MFC, Bugis A, Badawy N, Aboelghar HM, Hamouda TE and Jamjoom. AA: Staged Surgical Palliation for HLHS in a Girl with Severe Factor X Deficiency. Thorac Cardiovasc Surg Rep 2018; 7:e12-e15.

7. 7-Ewila H, Eltigani A, Abdelaziz A, Badr A, Kindawi A and Ahmed ElmahrouK: Preoperative hemoglobin concentration as an independent predictor for outcome after coronary artery bypass grafting. Journal of the Egyptian Society of Cardio-thoracic Surgery 24 (2016) 265-69 


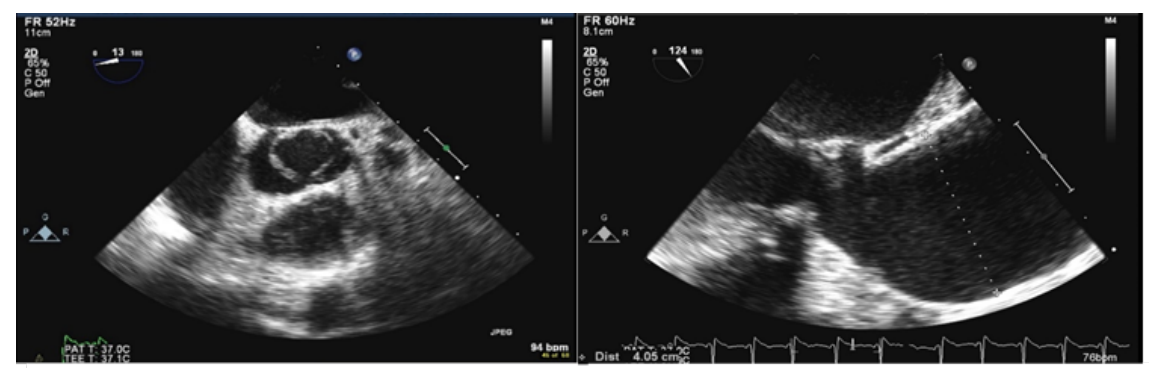

a.

b.

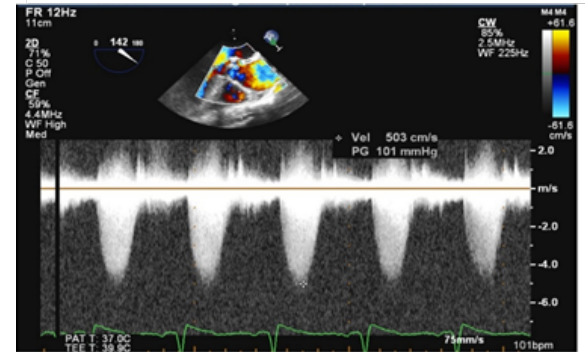

c.

Figure 1 Transesophageal echocardiogram demonstraitung. (a) Bicuspid aortic valve. (b) The aortic root. (c) With Color Doppler demonstrating a severe aortic stenosis

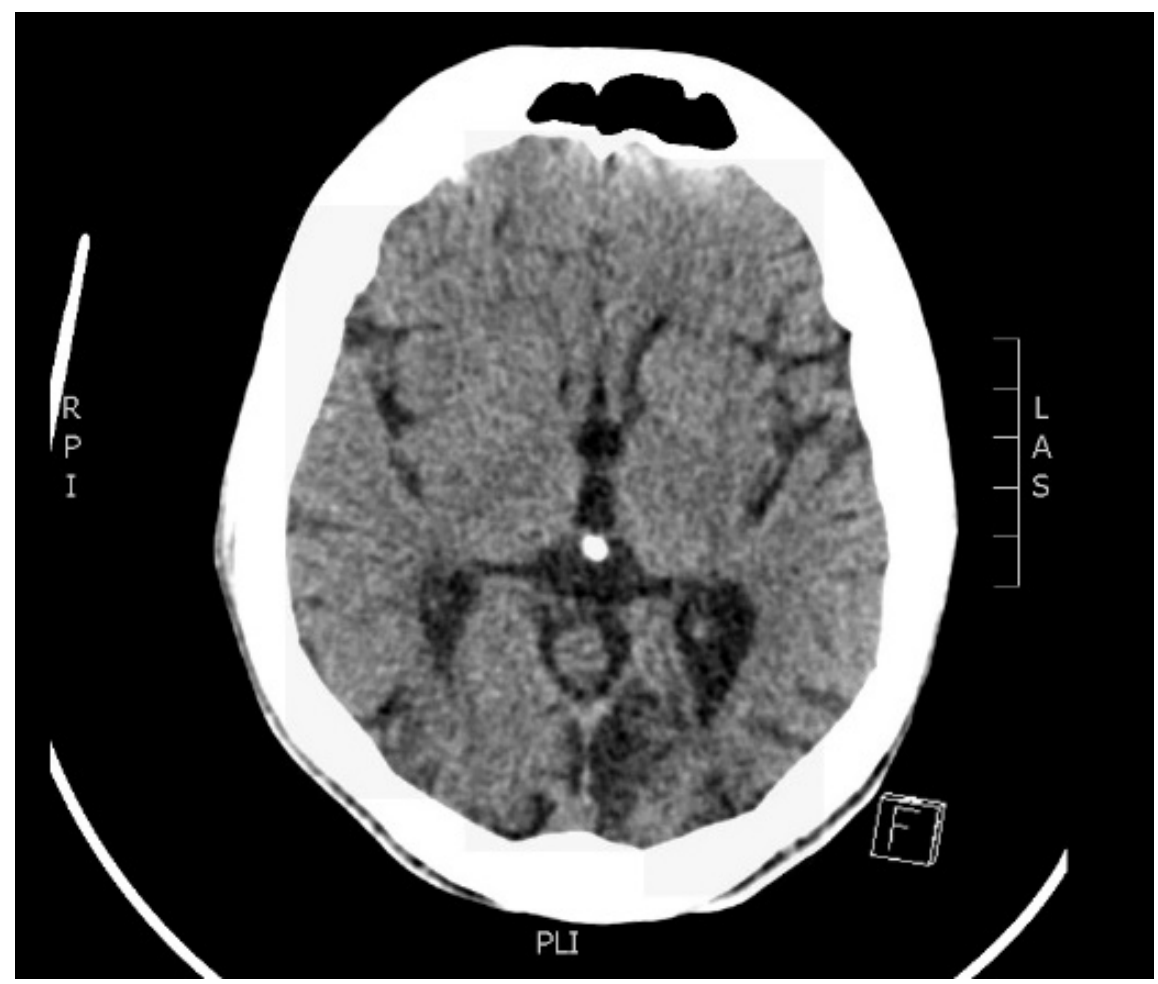

Figure 2 - CT scan demonstrates multiple old infractions mainly seen in the occipital lobe, posterior limb of the right internal capsule and the corona radiata/centrum semiovale with encephalomalacia changes. 


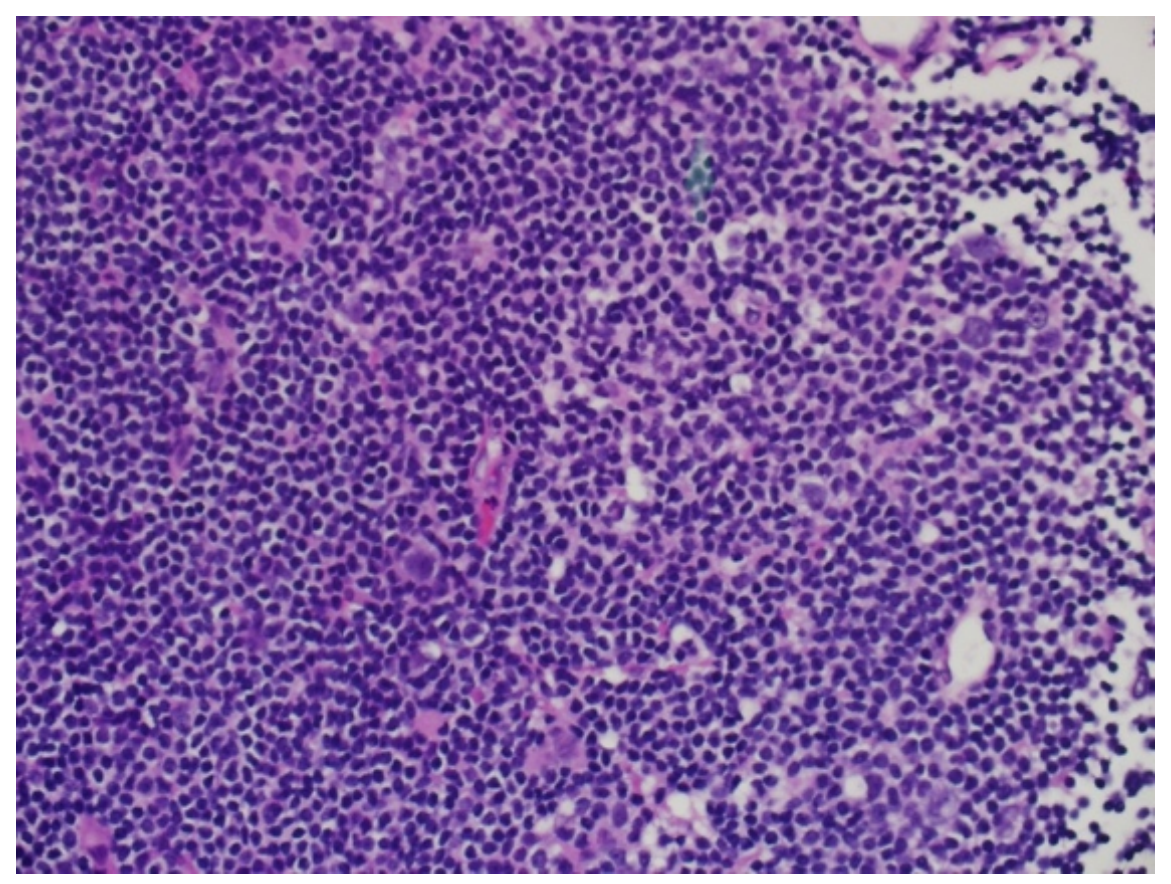

Figure 3 - Microscopic view demonstrating a scattered large a typical cells in the background of small lymphocytes, H\&E 100x

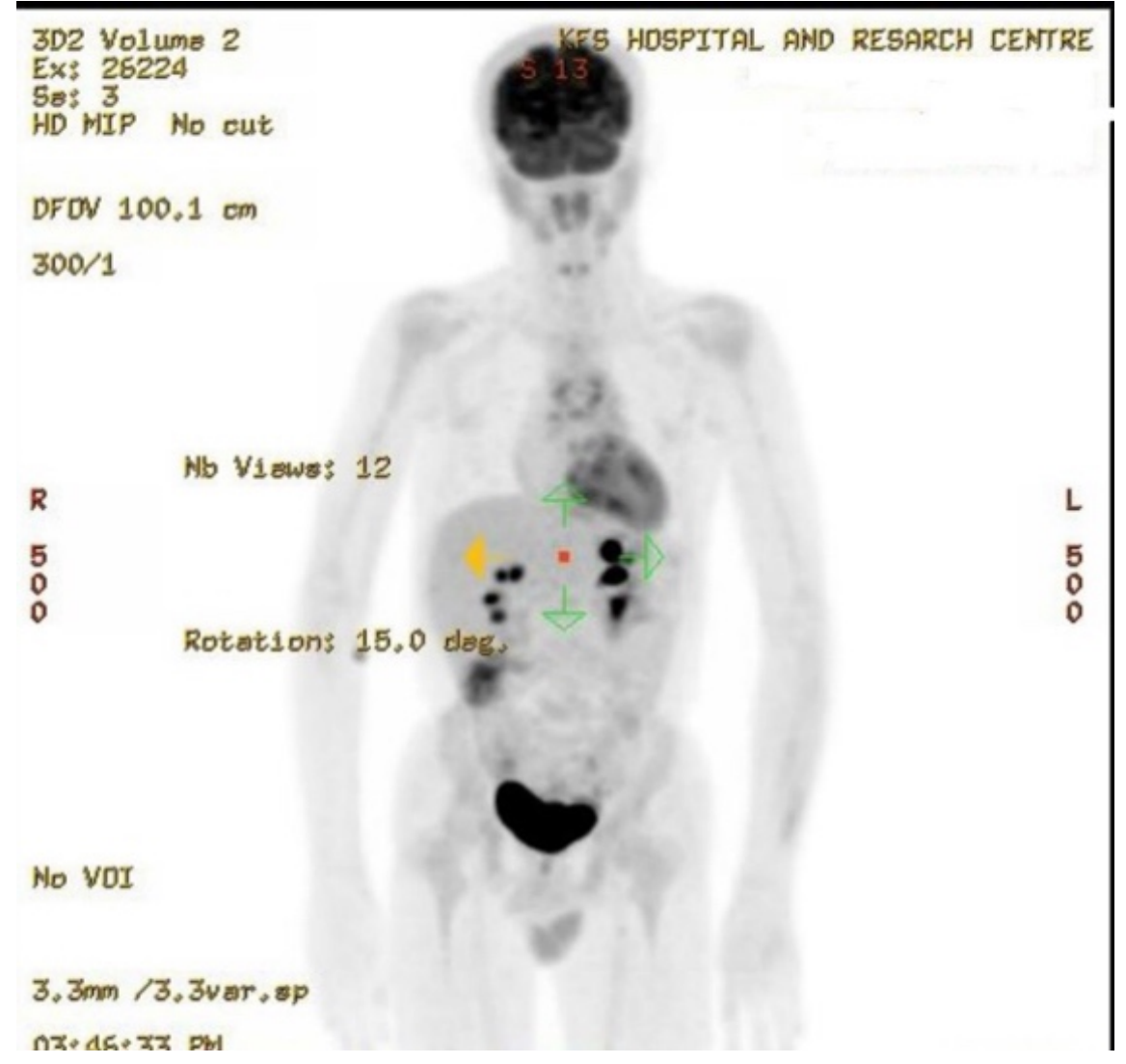


Figure 4 - There are multiple FDG avid enlarged lymph nodes seen in the left inguinal, obturator and external iliac lymph node chain. The largest measures around $3.2 \mathrm{~cm}$ (SUV max 15). 